Technical note

Subject Category: Enzymatic assays and analysis

\title{
Establishment of potent and specific synthetic substrate for dipeptidyl-peptidase 7
}

Takayuki K. Nemoto*, Toshio Ono, Yuko Ohara-Nemoto

Department of Oral Molecular Biology, Course of Medical and Dental Sciences, Nagasaki University Graduate School of Biomedical Sciences, Nagasaki 852-8588, Japan

*Corresponding author. Department of Oral Molecular Biology, Nagasaki University Graduate School of Biomedical Sciences, 1-7-1 Sakamoto, Nagasaki 852-8588, Japan. Email address: tnemoto@nagasaki-u.ac.jp (T. K. Nemoto) 


\section{ABSTRACT}

Bacterial dipeptidyl-peptidase (DPP) 7 liberates a dipeptide with a preference for aliphatic and aromatic penultimate residues from the N-terminus. Although synthetic substrates are useful for activity measurements, those currently used are problematic, because they are more efficiently degraded by DPP5. We here aimed to develop a potent and specific substrate and found that the $k_{\text {cat }} / K_{\mathrm{m}}$ value for Phe-Met-methylcoumaryl-7-amide (MCA) $\left(41.40 \pm 0.83 \mu \mathrm{M}^{-1} \mathrm{~s}^{-1}\right)$ was highest compared to Met-Leu-, Leu-Leu-, and Phe-Leu-MCA $\left(1.06-3.77 \mu \mathrm{M}^{-1} \mathrm{~s}^{-1}\right)$. Its hydrolyzing activity was abrogated in a Porphyromonas gingivalis dpp7-knockout strain. Conclusively, we propose Phe-Met-MCA as an ideal synthetic substrate for DPP7.

Keywords:

Dipeptidyl-peptidase 5, Dipeptidyl-peptidase 7, Periodontopathic bacteria, Porphyromonas gingivalis, Substrate specificity

Conflicts of interest: none. 


\section{Introduction}

Dipeptidyl-peptidases (DPPs) are exopeptidases that liberate a dipeptide from a polypeptide with an unmodified N-terminus. Based on the substrate specificities and enzymatic properties, 11 members of DPPs have been identified [1]. Among them, DPP4 $[2,3]$, DPP5 [4, 5], bacterial [6] and mammalian [7, 8] DPP7, and DPP11 [9] are serine peptidases, and DPP $8-10$ are allocated for subtypes of DPP4. DPP7 was allocated for two distinct DPPs, bacterial DPP7 [6] and mammalian one [7]. Bacterial DPP7 was first discovered in Porphyromonas gingivalis and is distributed solely in bacterial species [6]. On the other hand, human DPP7 is identical to DPPII and QPP/quiescent cell proline dipeptidase [8]. Although DPPs generally share distinct substrate specificities at the penultimate position from the N-terminus ( $\mathrm{P} 1$ position), the $\mathrm{P} 1$-position specificity of DPP5 partially overlaps with that of bacterial DPP7, as DPP5 prefers Ala and aliphatic residues $[4,5]$, and bacterial DPP7 prefers aliphatic and aromatic ones [8,9]. Nevertheless, there is no amino acid sequence similarity between DPP5 and bacterial DPP7 (11.7\%), and the topologies of the three essential amino acid residues of serine peptidases are completely distinct; $\mathrm{Ser}^{542}$, $\mathrm{Asp}^{627}$, and His ${ }^{659}$ in DPP5 [5], and His ${ }^{89}, \mathrm{Asp}^{225}$, and $\mathrm{Ser}^{648}$ in DPP7 [11].

Porphyromonas gingivalis, an asaccharolytic periodontopathic bacterium, expresses all these serine peptidase family DPPs in periplasmic space, where they manage efficient production of nutritious dipeptides being incorporated into the organism [12]. In addition to their necessity for bacterial metabolism, DPPs may be associated with physiological events through degradation of bioactive peptides in humans. For example, it is well known that mammalian DPP4 is a key enzyme involved in regulation of blood glucose levels via degradation of incretin peptides, glucagon-like peptide 1 (GLP-1) and gastric inhibitory polypeptide (GIP) $[13,14]$. As for the host-parasite relationship, we recently demonstrated that periodontopathic bacterial DPP4 hydrolyzes GLP-1 and GIP as does mammalian DPP4, resulting in enhancement and prolongation of postprandial hyperglycemia [15]. In addition, DPP7 in a canine oral bacterium, Capnocytophaga canimorsus, degrades Factor X and retard blood coagulation [16]. Thus, it is of particular interest to elucidate whether DPP7 in human indigenous bacteria exhibits similar physiological effects. To examine these 
issues, development of a synthetic substrate for DPP7 to separately quantitate DPP7 and DPP5 activities in bacteria as well as clinical specimens is eagerly anticipated.

We previously found that $P$. gingivalis DPP7 prefers hydrophobic residues at the P2 as well as P1 position [10], while DPP5 no apparent specificity at the P2 position [5]. Hence, DPP7 more potently hydrolyzes Met-Leu-methylcoumaryl-7-amide (MCA) than Lys-Ala-MCA, despite the fact that Met-Leu-MCA is more efficiently hydrolyzed by DPP5 than DPP7. From the viewpoint of maximum utilization of the P2-position hydrophobic preference of DPP7, the combination of a hydrophobic P2 residue and non-hydrophobic P1 residue, such as Leu-Arg-, Leu-Gln-, and Leu-Glu-MCA, resulted in highly DPP7-specific hydrolysis [5]. However, the hydrolyzing efficiency of DPP7 for these substrates was substantially lower as compared to that for Met-Leu-MCA. To address this, the present study aimed to develop a synthetic dipeptidyl fluorescent substrate that is efficiently and specifically hydrolyzed by DPP7.

\section{Materials and methods}

\subsection{Materials}

Lys-Phe-, Lys-Leu-, Lys-Val-, Lys-Met-, Phe-Leu-, Leu-Leu-, Phe-Met-MCA and dipeptides, Phe-Met, Leu-Asp, and Arg-Asp, were synthesized by Scrum (Tokyo, Japan). Other peptidyl MCA were purchased from the Peptide Institute (Osaka, Japan) and Bachem (Bubendorf, Switzerland). $\alpha$-Chymotrypsin from bovine pancreas (type II), Gly-Gln, and Gly-Glu were purchased from Merck (NJ, USA).

\subsection{Methods}

\subsubsection{Bacterial culture}

P. gingivalis strain ATCC 33277, and its derivatives NDP300 (dpp5::[ermF ermAM]) and NDP400 ( $\operatorname{dpp} 7::$ tet $Q)$ [5] were grown under an anaerobic condition $\left(80 \% \mathrm{~N}_{2}, 10 \% \mathrm{CO}_{2}\right.$, $10 \% \mathrm{H}_{2}$ ) in Anaerobic Bacterial Culture Medium broth (Eiken Chemical, Tokyo, Japan), 
supplemented with $0.5 \mu \mathrm{g} / \mathrm{ml}$ of menadione with or without appropriate antibiotics (10 $\mu \mathrm{g} / \mathrm{ml}$ of erythromycin or $0.7 \mu \mathrm{g} / \mathrm{ml}$ of tetracycline). Bacterial cells were harvested and washed with phosphate-buffered saline (PBS) at $\mathrm{pH}$ 7.4. The resulting cell pellets were re-suspended in PBS with absorbance adjusted at $600 \mathrm{~nm}$ to 2, then subjected to a DPP assay.

\subsubsection{Expression and purification of recombinant proteins}

The method for construction of plasmids, as well as the expressions of $P$. gingivalis DPP5 and DPP7 have been reported [5, 9]. Recombinant forms of P. gingivalis DPP3 [5], DPP4 [17], DPP11 [9], and acylpeptide oligopeptidase (AOP) [18] were purified as previously described.

\subsubsection{Measurement of peptidase activity}

Assays of peptidase activity were started by adding recombinant proteins (1 ng - 1 $\mu \mathrm{g})$ or $5 \mu \mathrm{l}$ of cell suspension to a reaction mixture $(200 \mu \mathrm{l})$ composed of $50 \mathrm{mM}$ sodium phosphate buffer ( $\mathrm{pH}$ 7.0), $5 \mathrm{mM}$ EDTA, and $20 \mu \mathrm{M}$ dipeptidyl MCA. After 30 min at 37 ${ }^{\circ} \mathrm{C}$, fluorescence intensity was measured with excitation at $380 \mathrm{~nm}$ and emission at $460 \mathrm{~nm}$. Activity of DPP5 was measured in the presence of $0.1 \mathrm{M} \mathrm{NaCl}$, and enzymatic parameters were calculated as described previously [5]. Experiments were conducted in triplicate and at least three times separately.

\section{Results}

\subsection{P1- and P2-position substrate specificities of $\mathrm{P}$. gingivalis DPP5 and DPP7}

First, to examine effects of the P1-position residue based on hydrophobicity, enzymatic activities were measured with five dipeptidyl MCA substrates with Lys at the P2 position (Fig. 1). DPP5 most efficiently hydrolyzed Lys-Ala-MCA, followed by Lys-Leu- and Lys-Phe-MCA, indicating an Ala preference at the P1 position. On the other hand, the order of hydrolysis efficiency was altered in DPP7, in which Lys-Met-MCA was 
most efficiently hydrolyzed by DPP7. Moreover, specific activities of DPP7 for these substrates were approximately one-tenth of those of DPP5, presumably because of the presence of the unwilling $\mathrm{P} 2$ residue. These results demonstrate that $\mathrm{P} 1$ Met [hydrophobicity index (H.I.) = 74] [19] was most preferred by DPP7 beyond its lower H.I. value compared to Phe $($ H.I.=100), Leu $($ H.I.=97), and Val $($ H.I.=76).

Next, we examined the effects of the P2-position residues with six dipeptidyl MCA substrates carrying Phe $($ H.I. $=100)$ or Leu $($ H.I. $=97)$ as a hydrophobic residue at the P1 position. There was no apparent tendency shown by DPP5 between hydrophobicity of the P2 residue and efficiency. In contrast, the efficiency of hydrolysis by DPP7 was strictly dependent on P2-position hydrophobicity, because it more efficiently hydrolyzed Gly-Phe-MCA than Lys-Phe-MCA. Furthermore, among the four dipeptidyl MCA substrates with P1 Leu, DPP7 most efficiently hydrolyzed Phe-Leu-MCA, followed by Leu-Leu- and Met-Leu-MCA. Accordingly, DPP7 activity was strictly dependent on the H.I. of the P2-position residues. These findings suggest that Met was the best at the P1 position beyond its moderate H.I. of 74, while the most hydrophobic residue Phe (H.I=100) was best at the $\mathrm{P} 2$ position.

\subsection{Potency of Phe-Met-MCA as DPP7 substrate}

Based on the data of Fig. 1, we newly synthesized Phe-Met-MCA and evaluated its potentials. Hydrolysis of Phe-Met-MCA by DPP7 was markedly higher as compared to hydrolyses of other substrates and all five substrates by DPP5 (Fig. 2). We determined the $k_{\text {cat }}\left(22,056 \pm 1,529 \mathrm{~s}^{-1}\right)$ and $K_{\mathrm{m}}(51.1 \pm 4.5 \mu \mathrm{M})$ of DPP7 for Phe-Met-MCA, and found that the resulting $k_{\text {cat }} / K_{\mathrm{m}}$ value $\left(41.40 \pm 0.83 \mu \mathrm{M}^{-1} \mathrm{~s}^{-1}\right)$ was substantially higher than those of Met-Leu-MCA $\left(1.06 \pm 0.25 \mu \mathrm{M}^{-1} \mathrm{~s}^{-1}\right)$, Leu-Leu- $\left(3.74 \pm 0.11 \mu \mathrm{M}^{-1} \mathrm{~s}^{-1}\right)$ and Phe-Leu-MCA $\left(1.53 \pm 0.06 \mu \mathrm{M}^{-1} \mathrm{~s}^{-1}\right)$. The reason for these differences was mainly due to the highest $k_{\text {cat }}$ value for Phe-Met-MCA as compared to Met-Leu- $\left(39.4 \pm 7.9 \mathrm{~s}^{-1}\right)$, Leu-Leu- $\left(75.4 \pm 0.2 \mathrm{~s}^{-1}\right)$, and Phe-Leu-MCA $\left(204.4 \pm 25.4 \mathrm{~s}^{-1}\right)$.

We examined whether Phe-Met-MCA is hydrolyzed by other peptidases. When recombinant forms of $P$. gingivalis DPP3, DPP4, DPP5, DPP7, DPP11, and AOP were incubated with this substrate, DPP5 showed faint activity and no hydrolysis was observed 
with the other exopeptidases (Fig. 2). In addition, we confirmed that $\alpha$-chymotrypsin, an endopeptidase with a preference for aliphatic P1 residues, did not exhibit activity towards Phe-Met-MCA (data not shown). Finally, we investigated the hydrolysis of Lys-Ala-, Phe-Met-, and Leu-Asp-MCA, i.e., specific substrates for DPP5, DPP7, and DPP11, respectively, by $P$. gingivalis wild-type, and NDP300 ( $\Delta d p p 5)$ and NDP400 ( $\Delta d p p 7)$ cells. The hydrolyses of Phe-Met-MCA did not change in NDP300, while significantly reduced to $15.8 \%$ in NDP400 as compared to the wild type.

\subsection{Inhibition of DPP7 activity by Phe-Met}

We previously reported that the dipeptides Leu-Asp, Arg-Asp, and Arg-Glu inhibited DPP11 activity in this order [20]. Hence, we examined whether the dipeptide Phe-Met inhibits DPP7 activity in comparison with Leu-Asp, Arg-Asp, Gly-Gln, and Gly-Glu. When measured at $5 \mu \mathrm{M}$ of Phe-Met-MCA. The activity was decreased to $83.5 \%$ by addition of 10-fold molar excess of Phe-Met, but not by others, which indicated that Phe-Met competitively inhibits the peptidase activity.

\section{Discussion}

The present study demonstrates that Phe-Met-MCA (or Phe-Met- $p$-nitroaniline for a chromogenic assay) is an ideal synthetic dipeptidyl substrate with a fluorescent group for measurement of DPP7 activity. Authentic dipeptidyl substrates for DPP3 (Arg-Arg-MCA), DPP4 (Gly-Pro-MCA), DPP5 (Lys-Ala-MCA), and DPP11 (Leu-Asp/Glu-MCA) have already been established, thus establishment of a DPP7 substrate as well should greatly facilitate studies of DPPs in periodontopathic oral as well as enteric bacteria, which express these DPPs.

In addition to the role of DPP7 in bacterial metabolism, we consider it important to search for physiological substrates. We recently reported that DPP4 in human periodontopathic bacteria, i.e., P. gingivalis, Tannerella forsythia, and Prevotella intermedia, hydrolyzed the incretin peptides GLP-1 and GIP, resulting in decreased plasma 
insulin concentrations, while it inversely raised the level of postprandial hyperglycemia and retarded the recovery of blood glucose levels in a mouse model [15]. Furthermore, Hack et al. [16] reported that DPP7 from C. canimorsus degraded coagulation Factor X, thereby inducing bleeding tendency. Since identification of in vivo targets of DPPs is the basis for elucidation of novel physiological and pathological roles in hosts-indigenous bacterial relationships, the establishment of the DPP7 substrate Phe-Met-MCA should facilitate such studies.

We recently showed that DPP11 captures substrate peptides strongly at the P1 and P2 positions, and weakly at the $\mathrm{P} 1$ ' position, while the $\mathrm{P} 2$ '-position residue was found freely present [20]. Consistent with those findings, Leu-Asp was revealed to have a high potential for inhibition of DPP11 activity. A similar finding that Phe-Met inhibits DPP7 activity should be useful for development of potent DPP7 inhibitors.

\section{Acknowledgments}

This work was supported by JSPS KAKENHI (grant numbers JP15K11047 and JP16K11481).

\section{References}

[1] N.D. Rawlings, M. Waller, A.J. Barrett, A. Bateman, MEROPS: the database of proteolytic enzymes, their substrates and inhibitors. Nucleic Acids Res. 42 (2014) D503-D509.

[2] Y. Misumi, Y. Hayashi, F. Arakawa, Y. Ikehara, Molecular cloning and sequence analysis of human dipeptidyl peptidase IV, a serine proteinase on the cell surface, Biochim. Biophys. Acta 1131 (1992) 333-336.

[3] A. Banbula, M. Bugno, J. Goldstein, J. Yen, D. Nelson, J. Travis, J. Potempa, Emerging family of proline-specific peptidases of Porphyromonas gingivalis: purification and characterization of serine dipeptidyl peptidase, a structural and 
functional homologue of mammalian prolyl dipeptidyl peptidase IV, Infect. Immun. 68 (2000) 1176-1182.

[4] A. Beauvais, M. Monod, J.P. Debeaupuis, M. Diaquin, H. Kobayashi, J.P. Latge, Biochemical and antigenic characterization of a new dipeptidyl-peptidase isolated from Aspergillus fumigatus, J. Biol. Chem. 272 (1997) 6238-6244.

[5] Y. Ohara-Nemoto, S.M. Rouf, M. Naito, A. Yanase, F. Tetsuo, T. Ono, T. Kobayakawa, Y. Shimoyama, S. Kimura, K. Nakayama, K. Saiki, K. Konishi, T.K. Nemoto, Identification and characterization of prokaryotic dipeptidyl-peptidase 5 from Porphyromonas gingivalis, J. Biol. Chem. 289 (2014) 5436-5448.

[6] A. Banbula, J. Yen, A. Oleksy, P. Mak, M. Bugno, J. Travis, J. Potempa, Porphyromonas gingivalis DPP-7 represents a novel type of dipeptidylpeptidase, J. Biol. Chem. 276 (2001) 6299-6305.

[7] J.K. McDonald, T.J. Reilly, B.B. Zeitman, S. Ellis, Dipeptidyl arylamidase II of the pituitary. Properties of lysylalanyl- $\beta$-naphthylamide hydrolysis: inhibition by cations, distribution in tissues, and subcellular localization. J. Biol. Chem. 243 (1968) 2028-2037.

[8] M.B. Maes, A.M. Lambeir, K. Gilany, K. Senten, P. Van der Veken, B. Leiting, K, Augustyns, S. Scharpe, I. De Meester, Kinetic investigation of human dipeptidyl peptidase II (DPPII)-mediated hydrolysis of dipeptide derivatives and its identification as quiescent cell proline dipeptidase (QPP)/dipeptidyl peptidase 7 (DPP7). Biochem. J. 386 (2005) 315-324.

[9] Y. Ohara-Nemoto, Y. Shimoyama, S. Kimura, A. Kon, H. Haraga, T. Ono, T.K. Nemoto, Asp- and Glu-specific novel dipeptidyl peptidase 11 of Porphyromonas gingivalis ensures utilization of proteinaceous energy sources, J. Biol. Chem. 286 (2011) 38115-38127.

[10] S.M. Rouf, Y. Ohara-Nemoto, T. Ono, Y. Shimoyama, S. Kimura, T.K. Nemoto, Phenylalanine664 of dipeptidyl peptidase (DPP) 7 and phenylalanine671 of DPP11 mediate preference for P2-position hydrophobic residues of a substrate, FEBS Open Bio. 3 (2013) 177-181.

[11] Y. Sakamoto, Y. Suzuki, I, Iizuka, C. Tateoka, S, Roppongi, M. Fujimoto K. Inaka, H. Tanaka, M. Masaki, K. Ohta, H. Okada, T. Nonaka, Y. Morikawa, K.T. Nakamura, W. 
Ogasawara, N. Tanaka, S46 peptidases are the first exopeptidases to be members of clan PA, Sci. Rep. 4 (2014) 4977. doi: 10.1038/srep04977.

[12] T.K. Nemoto, Y. Ohara-Nemoto, Exopeptidases and gingipains in Porphyromonas gingivalis as prerequisites for its amino acid metabolism, Jpn. Dental Sci. Rev. 52 (2016) 22-29.

[13] R. Mentlein, B. Gallwitz, W.E. Schmidt, Dipeptidyl-peptidase IV hydrolyses gastric inhibitory polypeptide, glucagon-like peptide-1(7-36)amide, peptide histidine methionine and is responsible for their degradation in human serum, Eur. J. Biochem. 214 (1993) 829-835.

[14] A.E. Weber, Dipeptidyl peptidase IV inhibitors for the treatment of diabetes, J. Med. Chem. 47 (2004) 4135-4141.

[15] Y. Ohara-Nemoto, M. Nakasato, Y. Shimoyama, T.T. Baba, T. Kobayakawa, T. Ono, T. Yaegashi, S. Kimura, T.K. Nemoto, Degradation of incretins and modulation of blood glucose levels by periodontopathic bacterial dipeptidyl peptidase 4 . Infect. Immun. 2017 doi: 10.1128/IAI.00277-17.

[16] K. Hack, F. Renzi, E. Hess, F. Lauber, J. Douxfils, J.M. Dogné, G.R. Cornelis, Inactivation of human coagulation factor $\mathrm{X}$ by a protease of the pathogen Capnocytophaga canimorsus. J. Thromb. Haemost. 15 (2017) 487-499.

[17] S.M. Rouf, Y. Ohara-Nemoto, T. Hoshino, T. Fujiwara, T. Ono, T.K. Nemoto, Discrimination based on Gly and Arg/Ser at Position 673 between dipeptidyl-peptidase (DPP) 7 and DPP11, widely distributed DPPs in pathogenic and environmental Gram-negative bacteria, Biochimie 95 (2013) 824-832.

[18] T.K. Nemoto, Y. Ohara-Nemoto, G.A. Bezerra, Y. Shimoyama, S. Kimura, A Porphyromonas gingivalis periplasmic novel exopeptidase, acylpeptidyl oligopeptidase, releases $\mathrm{N}$-acylated di- and tripeptides from oligopeptides. J. Biol. Chem. 291 (2016) 5913-5925.

[19] O.D. Monera, T.J. Sereda, N.E. Zhou, C.M. Kay, R.S. Hodges, Relationship of side chain hydrophobicity and $\alpha$-helical propensity on the stability of the single-stranded amphipathic $\alpha$-helix. J. Pept. Sci. 1 (1995) 319-329.

[20] G.A. Bezerra, Y. Ohara-Nemoto, I. Cornaciu, S. Fedosyuk, G. Hoffmann, A. Round, J.A. Márquez, T.K. Nemoto, K. Djinović-Carugo, Bacterial protease uses distinct thermodynamic signatures for substrate recognition, Sci. Rep. 7 (2017) 2848. 

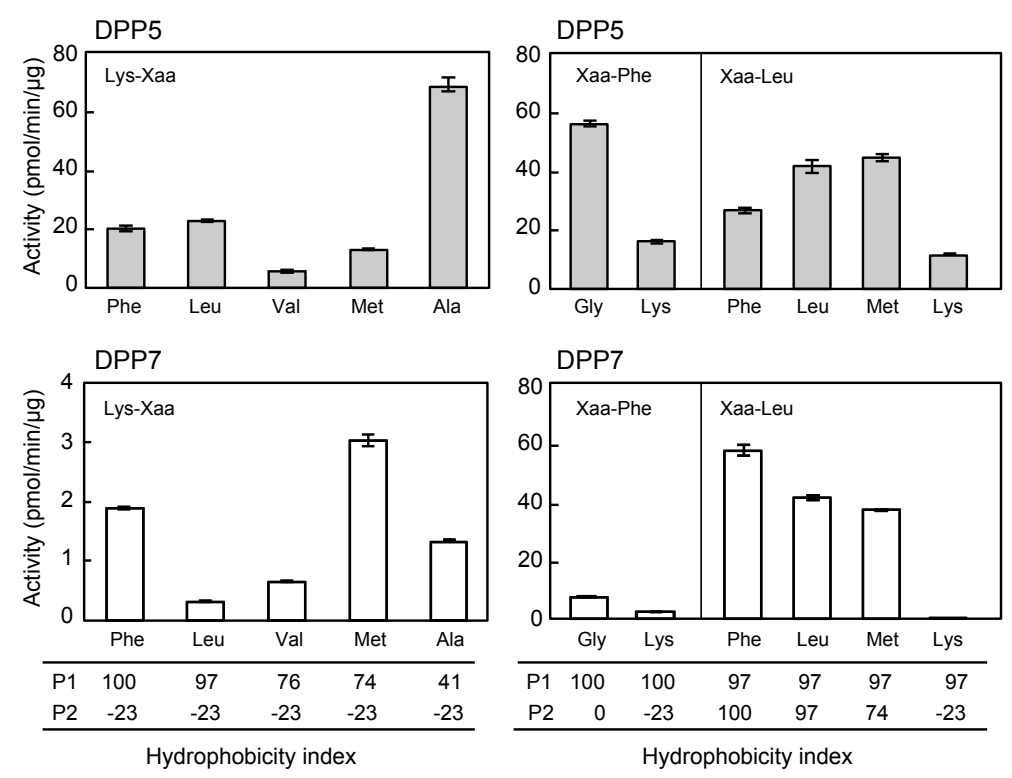

Fig. 1. Effects of P1- and P2-position residues of MCA substrates on activities of DPP5 and DPP7. Activities of DPP5 and DPP7 were determined with 5 dipeptidyl MCA containing Lys at the P2 position (left) and 6 dipeptidyl MCA containing Phe or Leu at the P1 position (right). Hydrophobicity indices of the P1- and P2-position residues are shown at the bottom. 
A

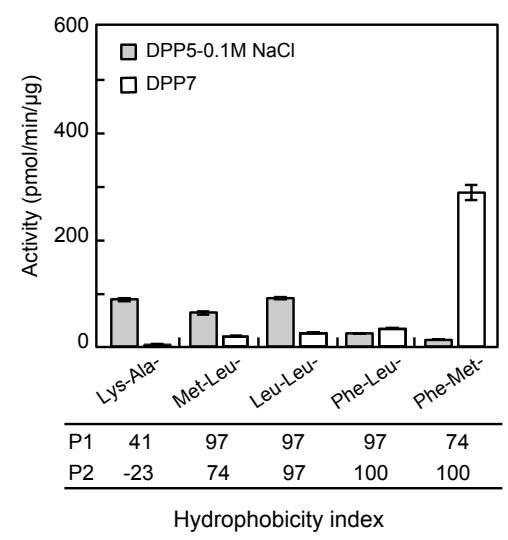

B

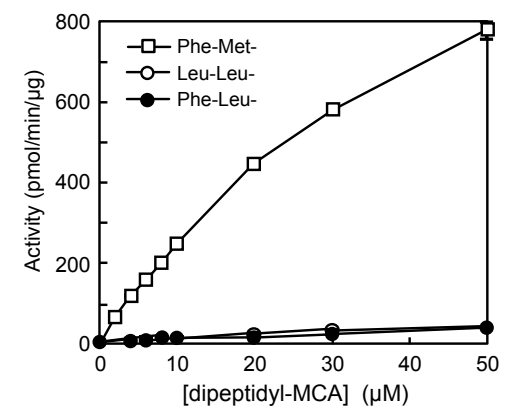

C

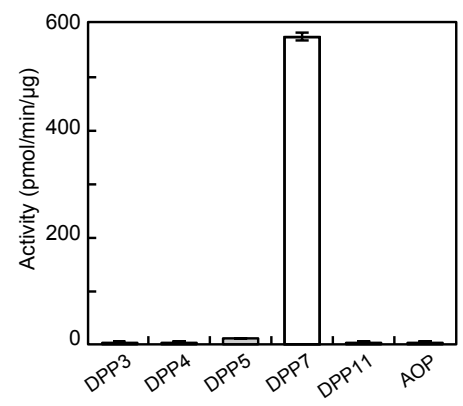

Fig. 2. Properties of potent substrates for DPP7. (A) Activities of DPP5 and DPP7 were compared using five dipeptidyl MCA. H.I. of the P1- and P2-position residues are shown at the bottom. (B) The activity of DPP7 was determined with $0-50 \mu \mathrm{M}$ of dipeptidyl MCA. (C) Hydrolysis for Phe-Met-MCA was determined using DPPs or AOP, which were active for appropriate substrates, i.e., DPP3 for Arg-Arg-MCA [5], DPP4 for Gly-Pro-MCA [10], DPP5 for Lys-Ala-MCA [5], DPP11 for Leu-Asp-MCA [9], and AOP for benzyloxycarbonyl-Lys-Met-MCA [18]. Values are shown as the mean \pm S.D. $(n=3)$. 